\title{
Amitriptyline enhances the central component of physiological tremor
}

\author{
J Raethjen, M R Lemke, M Lindemann, R Wenzelburger, P Krack, G Deuschl
}

\begin{abstract}
Objectives-Postural tremor is a regularly encountered side effect of amitriptyline which can be strong enough to cause discontinuation of therapy. The aim was to characterise amitriptyline induced tremor and to assess if the central or reflex component of physiological tremor was modulated by this drug.

Methods-The postural hand tremor was measured in 15 patients on a clinical rating scale, by power spectral analysis of accelerometer, forearm flexor, and extensor EMG before and after the beginning of amitriptyline treatment for major depression or chronic pain syndrome. A coherence analysis between flexor and extensor muscles on the same side was performed. Results-There was a clinically visible increase in postural tremor in a third of these patients. The tremor amplitude measured by accelerometer total power increased in every patient under amitriptyline. The EMG synchronisation as reflected by significant peaks in the flexor or extensor spectrum generally occurring at higher frequencies $(8-18 \mathrm{~Hz})$ than the accelerometric tremor frequencies (6-11 Hz) did not change. The number of patients with a significant flexor-extensor coherence in the $7-15 \mathrm{~Hz}$ range increased significantly under amitriptyline, the frequency bands of significant coherence corresponded with the EMG frequencies, and both were independent of changes to the hand's resonant frequency by added inertia.
\end{abstract}

Conclusions-An enhancement of postural tremor under amitriptyline is a common phenomenon although not always clinically apparent. The increase in EMG-EMG coherence indicates an increased common central drive to the motor units as its frequency is not influenced by peripheral resonance or reflex mechanisms. This is the first account of a drug induced enhancement of the central component of physiological tremor.

(F Neurol Neurosurg Psychiatry 2001;70:78-82)

Keywords: drug induced tremor; tremor physiology; central drive

The current view of drug induced tremors in general is that an increase in the gain of the muscle receptors and spinal reflex loops leads to an enhancement of the oscillations in peripheral physiological tremor. This has been studied, for instance, for $\beta$-adrenergic agents ${ }^{12}$ and thyroid hormones. ${ }^{3}$ However, many drugs that can induce tremor mainly act within the CNS. One example of such a drug is amitriptyline, ${ }^{45}$ which can cause a sometimes disabling postural tremor of the hands when used as an antidepressant drug or analgesic. Although this is a well known clinical phenomenon ${ }^{4-7}$ its physiological basis has never been studied. Although there are also peripheral side effects ${ }^{8}$ it cannot be inferred that the tremor found under amitriptyline shares the same peripheral mechanisms with the other so far described drug induced postural tremors. A second well established component of physiological tremor which is independent of the periphery and peripheral reflex loops ${ }^{9}$ has been termed "central" and was postulated to arise from an oscillator within the CNS. ${ }^{10}{ }^{11}$ It seems plausible to hypothesise an enhancement of this central component as the mechanism of postural tremors occurring under centrally acting drugs such as amitriptyline. However, amitriptyline induced tremor has never been analysed physiologically and it has never been shown, so far, that the central component of physiological tremor can be enhanced by drugs at all. We used accelerometry and EMG before and after amitriptyline intake to characterise this tremor and to define its relation to normal physiological tremor. It will be shown for the first time that in parallel to the increase in tremor amplitude a synchronisation of motor units between different muscles in the $7-15 \mathrm{~Hz}$ band develops indicating an enhancement of the common central rhythmic input that is the central component of physiological tremor.

Preliminary results of this study have been published in abstract form. ${ }^{12}$

\section{Methods}

PATIENTS

Fifteen patients (six male, nine female) were analysed before amitriptyline intake (T0), 1 week after the beginning of amitriptyline treatment (T1), and after the individually effective dose was reached (T2). In one of the patients the final dosage was reached already at $\mathrm{T} 1$ so the analysis at T2 was not performed. Fourteen of the patients were treated for major depression and one for chronic lower back pain. None of the patients had any other neurological signs or symptoms and none of the patients had signs or a family history of pathological tremor. All patients were either drug naive or underwent a 3 day wash out period without any medication before the start of amitriptyline treatment. The protocol was approved by the local ethics committee and each patient signed an informed consent form. 
DATA RECORDINGS

Postural tremor of the hand was recorded with uniaxial accelerometry and bipolar surface EMG with the hand held at a $0^{\circ}$ position and the forearm supported. This recording was repeated with an extra $500 \mathrm{~g}$ and $1000 \mathrm{~g}$ loading of the hands. The accelerometers were fixed to the dorsum of the hands on the distal part of the third metacarpal bone $9 \mathrm{~cm}$ away from the ulnar epicondylus of the wrist. EMG electrodes were fixed on the muscle bellies close to the motor points of the extensor carpi and flexor carpi ulnaris muscles about $2 \mathrm{~cm}$ apart. The sampling rate of the EMG and accelerometry was $800 \mathrm{~Hz}$. The EMG was band pass filtered between 50 and $400 \mathrm{~Hz}$. The recording duration was 30 seconds for each condition.

A clinical rating of the postural and action tremor on a scale between 0 and $3^{13}$ was performed including a disability item based on the history and a performance test measuring the amount of spilled water when pouring it from one test tube to another, ${ }^{14} 0$ indicating no subjective disability or no water spilled at all and 3 indicating severe disabling tremor not allowing the task to be completed.

DATA ANALYSIS

Autospectral analysis resulting in power spectra of all channels and cross spectral analysis between extensors and flexors of the same side including coherence spectra were performed using a data driven adaptive time series analysis algorithm. ${ }^{15}{ }^{16} \mathrm{~A}$ significant coherence indicates a linear correlation between the components of a multivariate process. Whenever two oscillations are coherent this implies common inputs to both oscillating structures. Possible myoelectrical cross talk between pairs of recorded muscles which might lead to an artefactually high coherence estimate could be detected in the cross correlation function by applying an algorithm described elsewhere ${ }^{17}$ and was excluded from further analysis. Only four recordings of the present study had to be excluded because of excessive cross talk. The peak frequencies of the EMG and accelerometer spectra were determined in the power spectra and the total power of the accelerometer spectra between 0 and $30 \mathrm{~Hz}$ served as a measure of the tremor amplitude.

As the number of cases was small and because of the pilot character of the study the robust non-parametric Friedman test was used to find statistically significant differences between the three different recording times (T0, $\mathrm{T} 1$, T2). When this was significant the Wilcoxon test was applied to analyse the difference between the recordings before amitriptyline (T0) and $\mathrm{T} 1$ and $\mathrm{T} 2$.

\section{Results}

The clinical tremor scores increased from T0 to $\mathrm{T} 1$ in four patients in the examination and disability item. Three patients exhibited some postural tremor clinically on T0 already, the tremor deteriorated from $\mathrm{T} 0$ to $\mathrm{T} 1$ in one of these patients only, and it remained clinically unchanged in the others. The highest tremor score found throughout the whole study was 2 indicating a moderate postural tremor. Seven patients did not show any postural tremor and did not develop tremor clinically under amitriptyline either. The mean clinical tremor score is displayed in fig $1 \mathrm{~A}$. There was only a slight increase of postural tremor and disability between T0 and T1 and only the scores for the tremor in the right hand and the disability of the left hand were significant. By contrast with the clinical score the total power was increased in every patient from $\mathrm{T} 0$ to $\mathrm{T} 1$ under all three recording conditions and remained stable or showed a slight decrease from $\mathrm{T} 1$ to $\mathrm{T} 2$. The average increase in total power is illustrated in fig $1 \mathrm{~B}$ for all recording conditions and both sides. This increase in total power from $\mathrm{T} 0$ to $\mathrm{T} 1$ and $\mathrm{T} 2$ was statistically significant $(p<0.05)$ in all cases except for the difference between T0 and T2 on the right under the unloaded postural recording condition. The accelerometer frequencies were in the range of normal physiological tremor between 6 and 12 $\mathrm{Hz}$ and decreased significantly when the weight loads of $500 \mathrm{~g}$ or $1000 \mathrm{~g}$ were fixed to the hands. The frequencies did not change under amitriptyline. The EMG total power did not change in the extensors or in the flexors under amitriptyline (fig $1 \mathrm{C}$ ). Only under the recording condition with a $1000 \mathrm{~g}$ weight and only on the right side did the flexor tp increase significantly from T0 to T1. The EMG spectra of both recorded muscles showed a significant spectral peak indicating rhythmic EMG activity in $50 \%$ to $70 \%$ of the patients. The proportion of recordings with a significant EMG peak did not change under amitriptyline. The peak frequencies were distributed in the $8-18 \mathrm{~Hz}$ band, which is clearly higher than the main tremor frequencies as measured by accelerometry. They were variable but remained unaffected by added weight and did not show a systematic change under amitriptyline.

An influence of the rhythmic EMG activity on the accelerometric tremor is indicated in the accelerometer spectrum by a significant peak at the EMG frequency. It was assessed for all patients, recording conditions and both sides whether such an EMG driven tremor became more common or prominent under amitriptyline treatment. Examples of accelerometer and EMG spectra from two patients on T0 and T1 are shown in fig $2 \mathrm{~A}$ and $\mathrm{B}$. In B the spectra of accelerometer and EMG showed narrow peaks on T1 which appeared at the same frequency, by contrast with the recording before the start of amitriptyline treatment on T0 not showing a significant accelerometer peak corresponding to the EMG frequency (fig 2 B). The other patient (fig $2 \mathrm{~A}$ ) showed significant EMG peaks in both muscles on $\mathrm{T} 1$ as well and there was an increase in power in the accelerometer spectrum at the EMG frequency from T0 to T1 but this did not produce a significant peak. The accelerometer peak remained at a much lower frequency. In most patients we found spectra similar to the example in fig $2 \mathrm{~A}$ and some of the patients did not show significant EMG peaks at all, even after amitriptyline intake. Only three of the patients showed a clear EMG driven accelerometer peak on $\mathrm{T} 1$ as in the example of fig $2 \mathrm{~B}$ and one of 

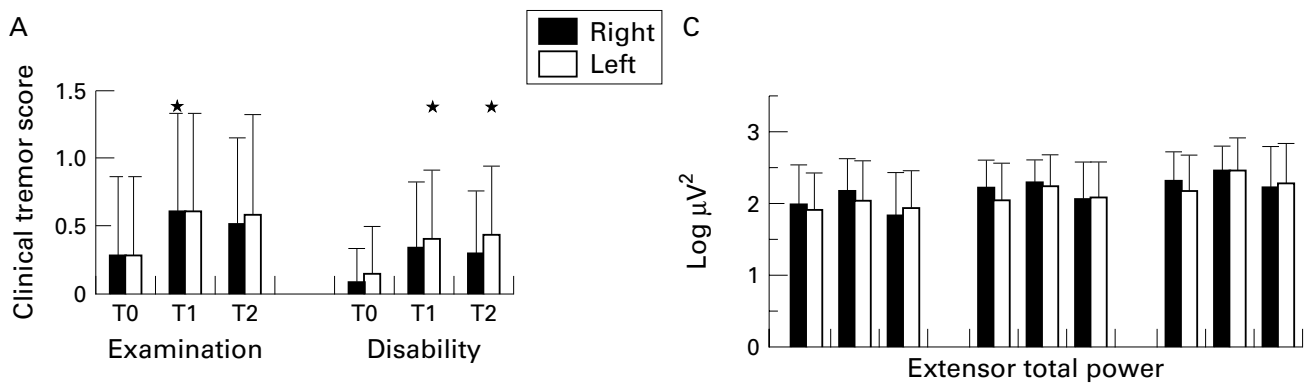

B

D
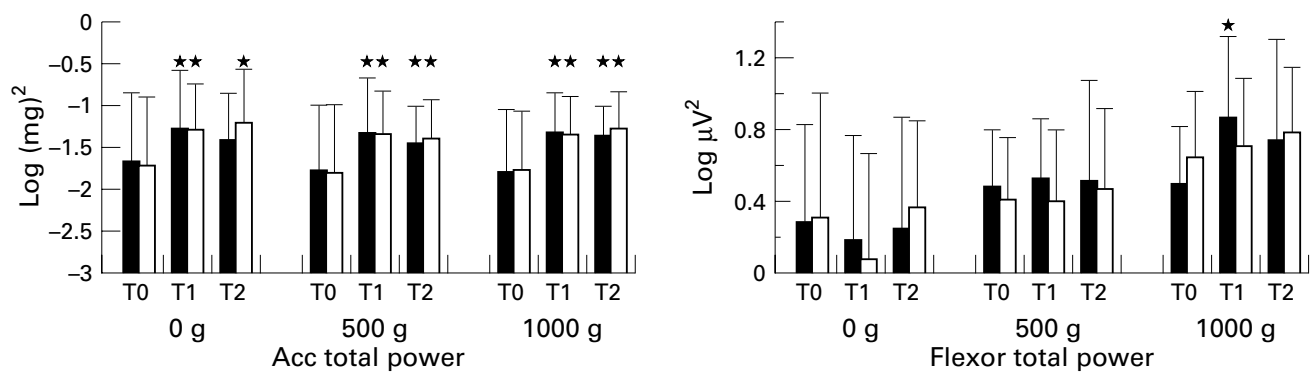

Figure 1 Tremor under amitritptyline. (A) Mean (SD) of the clinical tremor score is displayed for the examination and the disability items and for the right and left hand on the different days. There was a clear increase in the tremor score from TO to $T 1$ which was statistically significant $(p<0.05)$ only for the right side in the examination item and only for the left side in the disability items. On the single case level there was an increase in tremor score only for five of the 15 patients. (B) The increase in the accelerometer (Acc) total power as a measure of the tremor amplitude is shown for the three different recording conditions. The means (SD) of the logarithmically transformed total power values are displayed. The differences between T0 and T1 or T2 were statistically significant $(p<0.05)$. The total power increased in every patient. The total power of the extensor and flexor EMG spectra is shown in $C$ and D. There were no differences between T0, T1, and T2 Only for the flexors on the right and only under the $1000 \mathrm{~g}$ condition was there a significant increase from T0 to T1. $T 0=$ baseline; $T 1=1$ week after amitriptyline was started; $T 2=$ when the individually effective dose was reached. Statistical significant differences between TO and T1 or T2 are indicated by the asterisks $(p<0.05)$.

these patients had a significant accelerometer peak at the EMG frequency on T0 already. The group data on the occurrence of significant accelerometer peaks at the EMG frequency is displayed in fig $2 \mathrm{C}$. The percentage of such accelerometer peaks was variable between different recording conditions and the right and left side but there was no significant difference between the recordings before and after amitriptyline intake.

In the examples of fig $2 \mathrm{~A}$ and $\mathrm{B}$ the coherence spectra between extensor and flexor muscles are plotted at the bottom. In both cases we found a significant coherence in the $7-15 \mathrm{~Hz}$ band on T1 which corresponded to the EMG peak frequencies and was not present on T0. Such a development of EMG-EMG coherence under amitriptyline was a common effect which we found in $55 \%$ of the patients on $\mathrm{T} 1$ or T2 whereas it was only present in $8 \%-13 \%$ before amitriptyline treatment (T0). The percentage of significant EMG-EMG coherence between flexor and extensor on the same side is illustrated in fig $2 \mathrm{D}$ for all different recording conditions. The difference between $\mathrm{T} 0$ and $\mathrm{T} 1$ and $\mathrm{T} 2$ is clearly significant $(p<0.01)$. The phase relation between the coherent muscles was 0 in the vast majority of the patients indicating a rhythmic cocontraction between flexor and extensor. Only in one of the patients did we find a reciprocal alternating pattern. The frequency of significant coherence corresponded to the EMG frequencies which were in a much higher frequency range than the main tremor measured accelerometrically and did not change under added inertia. This independence of the amitriptyline induced EMG-EMG coherence from the mechanical main tremor frequency is demonstrated in the representative example in fig $2 \mathrm{~A}$ which shows for the unloaded recording condition that the frequency of significant coherence already occurs at an independent higher frequency band.

\section{Discussion}

Postural tremor under amitriptyline treatment is a commonly seen side effect which can become strong enough to be the reason for discontinuation of the therapy in psychiatric patients. ${ }^{78}$ However, tremor only occurs in some of the patients treated with amitriptyline and it sometimes improves in the course of the treatment. ${ }^{18}$ This is in line with our clinical rating, which only showed an increase in postural tremor in five out of 15 patients whereas in the remaining 10 patients there was no clinically visible tremor. But the accelerometry used in the present study showed an increase in tremor amplitude in all of our patients. This confirms that an enhancement of postural tremor is indeed a common effect of amitriptyline although often not seen clinically. What is the physiological basis of this tremor enhancement? One simple explanation for the increase in accelerometer power could be an increase of the extensor or flexor total power which is transmitted to the hand. We showed, however, that neither the flexor nor the extensor total power changed under amitriptyline. Another plausible reason could be a stronger tendency to rhythmic muscle activation. But the pro- 

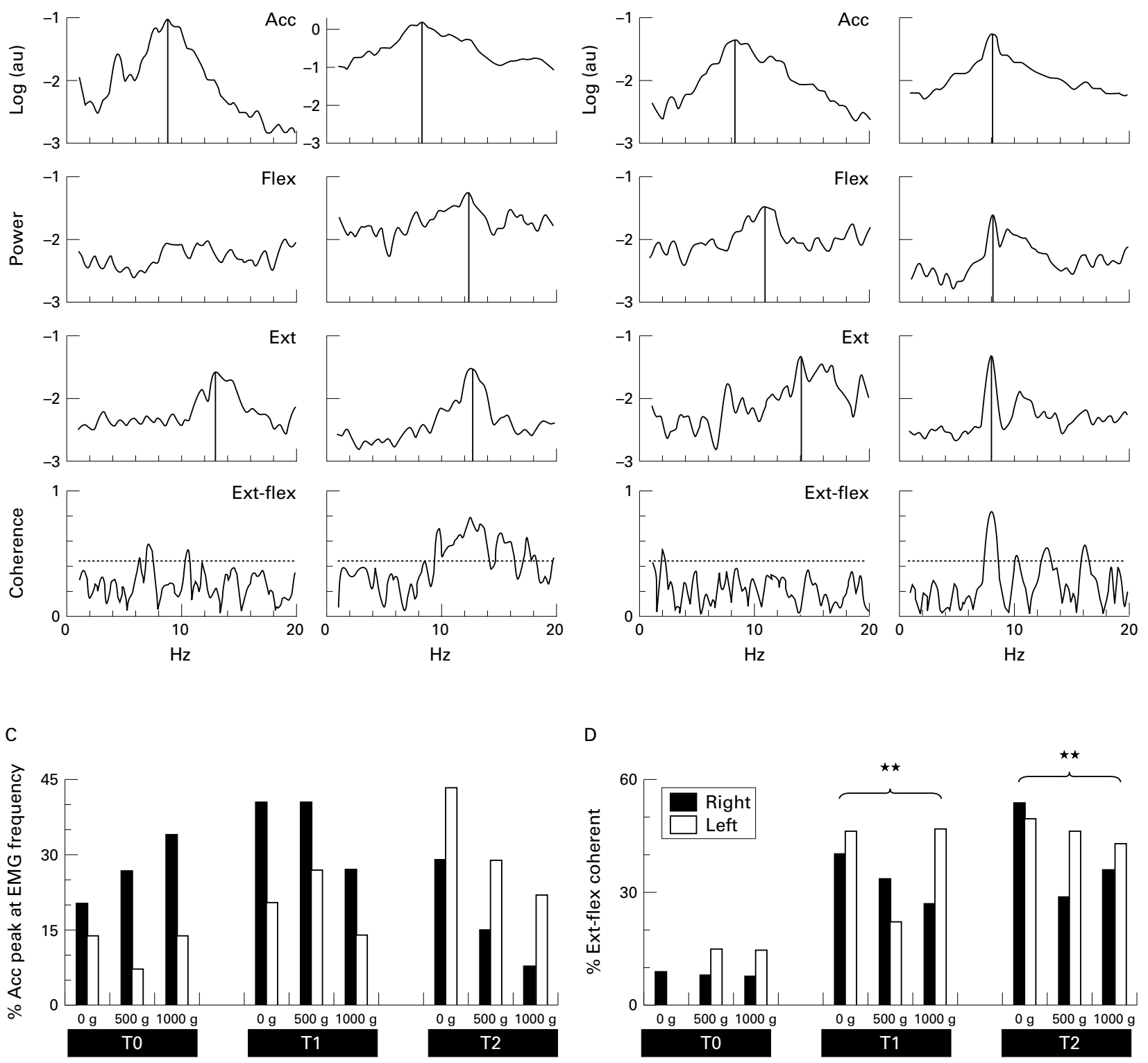

Figure 2 EMG synchronisation under amitriptyline. ( $A$ and B) Two examples of accelerometer, flexor, and extensor power spectra and extensor-flexor coherence spectra are shown for the unloaded postural condition before and after the initiation of amitriptyline treatment. The power spectra in $A$ show an increase of the total power under amitriptyline. There is a small but significant peak in the flexor spectrum which is not present before amitriptyline treatment and there is some increase in the power of the accelerometer spectrum in the range of the EMG frequencies. The accelerometric tremor peak remains at a much lower frequency than the EMG peaks. The main change can be seen in the coherence spectrum at the bottom. Although there was no significant coherence before amitriptyline the coherence spectrum clearly rises above the 5\% significance level, indicated by the horizontal line, at the EMG frequency on T1. This was the common picture under amitriptyline treatment. The example in B illustrates one case with a strong EMG synchronisation leading to an EMG driven tremor. In this case the band of significant coherence was narrower. (C) The percentage of patients with a significant accelerometer peak at the EMG frequency is displayed indicating an EMG synchronisation strong enough to cause a central tremor peak. These percentages were very variable but they did not change systematically under amitriptylin. (D) The percentage of patients with significant extensor-flexor coherence is shown for the different recording conditions and the left and right side for T0, T1, and T2. Taking all the recordings on the different days together the difference between TO and T1 or T2 is significant $(p<0.01)$.

portion of significant EMG peaks remained constant under amitriptyline and the influence of rhythmic EMG activity on the tremor spectrum was not enhanced either. However, the percentage of significant EMG synchronisation was extremely variable between different recording conditions which may be due to the poorer and possibly variable signal to noise ratio of the EMG data compared with the accelerometer recordings. The only change which clearly paralleled the increase in tremor amplitude was the higher proportion of coherent activation of antagonistic muscles in the
$7-15 \mathrm{~Hz}$ range. This higher proportion of coherence indicates stronger common inputs to the flexor and extensor muscles and could well cause or reflect a more effective rhythmic interaction between the antagonistic muscles. ${ }^{19}{ }^{20}$ In the case of a reciprocal alternating flexor-extensor interaction a higher tremor amplitude would clearly develop. But most of our patients showed a phase relation of 0 between flexors and extensors. Further studies are necessary to understand how this cocontraction leads to an increased mechanical tremor amplitude. Nevertheless, our results 
suggest that the development of this rhythmic coupling of antagonistic muscles causes the increase of accelerometer power.

As tremor induced by amitriptyline evolves from normal physiological tremor it seems likely that the increase in extensor-flexor coherence arises from the enhancement of one of its components. The two major components of physiological tremor are the peripheral mechanical reflex oscillation which is dependent on the hand's resonant frequency. ${ }^{3921}$ and the centrally driven component in the $7-14 \mathrm{~Hz}$ range. ${ }^{10} 11{ }^{22-24}$ The mechanical reflex component can be enhanced by an increased gain in the peripheral muscle receptors and spinal reflex loops. ${ }^{25}{ }^{26}$ If this was the mechanism of increased intermuscular coherence it would be expected that this significant coherence would occur at the main tremor frequency and change in parallel to the hand's resonant frequency. The central component of physiological tremor, which is centred around the $8-12 \mathrm{~Hz}$ range, ${ }^{27}$ has been postulated to originate from oscillators within the CNS as it is not influenced by peripheral changes such as added inertia that is independent of the the hand's resonant frequency. ${ }^{91124}$ An enhancement of this central component of physiological tremor would result in an increased common central rhythmic drive to the muscles leading to a significant coherence between different muscles at the frequency of this drive.$^{17}$ By contrast with the peripheral enhancement this frequency can be different from the hand's resonant frequency and will remain constant under added inertia. The frequency of the flexor-extensor coherence seen under amitriptyline in this study is indeed clearly higher than the main tremor frequency of the hand and remains in the 7-15 $\mathrm{Hz}$ range independently of peripheral changes. Thus our results clearly argue in favour of an enhancement of the central component of physiological tremor by amitriptyline, no matter if this central component originates in spinal or supraspinal pathways. To our knowledge this is the first physiological account of a drug induced enhancement of the central component of physiological tremor.

Such a drug induced amplification of rhythms in th $7-15 \mathrm{~Hz}$ range within the central motor system has two major implications. On the one hand it could be utilised to study the $8-12 \mathrm{~Hz}$ pulses governing slow movements ${ }^{28}$ which have recently been shown to also arise from the $\mathrm{CNS}^{29}{ }^{30}$ and most likely share similar mechanisms with the central component of physiological tremor. On the other hand it can be regarded as a model of a slowly evolving pathological postural tremor such as essential tremor and might elucidate the relation between physiological and pathological tremors which is still a matter of debate. ${ }^{1031} 32$ We hypothesise that the increase in centrally driven coupling between antagonistic muscles demonstrated in the present study is the basis on which certain pathological tremors may develop and it might be a first neurophysiological sign of an evolving tremor disorder.

We thank Ms M Witt for excellent technical assistance and Dr M Lauk, Freiburg, Germany, for designing the tremor program used in this study. This work was supported by the German Research Council (Deutsche Forschungsgemeinschaft, DFG, De 438,41). ML was supported by the German National Scholarship Foundation (Studienstiftung des Deutschen Volkes).

1 Marsden CD, Meadows JC. The effect of adrenaline on the contraction of human muscle. I Physiol (Lond) 1970;207:429-48.

2 Foley TH, Marsden CD, Owen DA. Evidence for a direct peripheral effect of adrenaline on physiological tremor in man. F Physiol (Lond) 1967;189:65P-6.

3 Hömberg V, Hefter H, Reiners K, et al. Differential effects of changes in mechanical limb properties on physiological and pathological tremor. F Neurol Neurosurg Psychiatry 1987;50:568-79.

4 Watanabe S, Yokoyama S, Kubo S, et al. A double-blind controlled study of clinical efficacy of maprotiline and amitriptyline in depression. Folia Psychiatr Neurol $\mathfrak{f p}_{p}$ 1978;32:1-31.

5 James B. A double-blind comparative clinical study of amoxapine and amitriptyline in depressed, hospitalised patients. N Z Med f 1982;95:391-3.

6 Petrie WM, Ban TA, Wilson WH, et al. Viloxazine in the treatment of endogenous depression. A standard (amitriptyline) controlled clinical study. Int Pharmacopsychiatry 1982;17:280-6.

7 Smith WT, Glaudin V, Panagides J, et al. Mirtazapine vs amitriptyline vs placebo in the treatment of major
depressive disorder. Psychopharmacol Bull 1990;26:191-6.

8 Chouinard G. A double-blind controlled clinical trial of fluoxetine and amitriptyline in the treatment of outpatients with major depressive disorder. F Clin Psychiatry 1985;46: 32-7.

9 Deuschl G, Krack P, Lauk M, et al. Clinical neurophysiology of tremor. F Clin Neurophysiol 1996;13:110-21.

10 Elble RJ. Mechanisms of physiological tremor and relationship to essential tremor. In: Findley LJ, Koller WC, eds. Handbook of tremor disorders. New York: Dekker, 1995:51-62.

11 Elble RJ. Central mechanisms of tremor. $\mathcal{f}$ Clin Neurophysiol 1996;13:133-44.

12 Raethjen J, Lemke MR, Wenzelburger R, et al. Amitriptyline enhances the central component of physiological tremor. Mov Disord 1998;13(suppl 3):134, P21.

13 Fahn S, Tolosa E, Marin C. Clinical rating scale for tremor. In: Jankovic J, Tolosa E, eds. Parkinson's disease and movement disorders. Baltimore: Williams and Wilkins, 1993:271-80.

14 Zimmermann R, Deuschl G, Hornig A, et al. Tremors in Parkinson's disease: symptom analysis and rating. Clin Neuropharmacol 1994;17:303-14.

15 Timmer J, Lauk M, Deuschl G. Quantitative analysis of tremor time series. Electroencephalogr Clin Neurophysiol 1996;101:461-8.

16 Lauk M, Timmer J, Lücking $\mathrm{CH}$, et al. A software for recording and analysis of human tremor. Comput Methods Programs Biomed 1999;60:65-77.

17 Raethjen J, Lindemann M, Schmaljohann H, et al. Multiple oscillators are causing parkinsonian and essential tremor. Mov Disord 2000;15:84-94

18 Fruensgaard K, Hansen CE, Korsgaard S, et al. Amoxapine versus amitriptyline in endogenous depression. A doubleblind study. Acta Psychiatr Scand 1979;59:502-8.

19 Timmer J, Lauk M, Pfleger W, et al. Cross-spectral analysis of physiological tremor and muscle activity. I. Theory and application to unsynchronized electromyogram. Biol Cybern 1998;78:349-57.

20 Amjad AM, Halliday DM, Rosenberg JR, et al. An extended difference of coherence test for comparing and combining several independent coherence estimates: theory and application to the study of motor units and physiological tremor. cation to the study of motor units and
$\exists$ Neurosci Methods 1997;73:69-79.

21 Stiles RN, Pozos RS. A mechanical-reflex oscillator hypothesis for parkinsonian hand tremor. $\mathcal{F}$ Appl Physiol 1976;40: esis for

22 Halliday DM, Conway BA, Farmer SF, et al. Loadindependent contributions from motor-unit synchronization to human physiological tremor. I Neurophysiol 1999;82:664-75.

23 Hallett M. Overview of human tremor physiology. Mov Disord 1998;13:43-8.

24 Elble RJ, Randall JE. Motor-unit activity responsible for 8-12 Hz component of human physiological finger tremor. 7 Neurophysiol 1976;39:370-83.

25 Rack PMH. Mechanical and reflex factors in human tremor. In: Desmedt JE, ed. Progess in clinical neurophysiology. Basel: Karger, 1978:17-27

26 Hagbarth KE, Young RR. Participation of the stretch reflex in human physiological tremor. Brain 1979;102:509-26.

27 Lippold OC. Oscillation in the stretch reflex arc and the origin of the rhythmical, 8-12 C-S component of physiologigin of the rhythmical, 8-12 C-S component of

28 Vallbo AB, Wessberg J. Organization of motor output in slow finger movements in man. $\mathcal{F}$ Physiol (Lond) 1993;469: 673-91.

29 Wessberg J, Vallbo AB. Pulsatile motor output in human finger movements is not dependent on the stretch reflex. $f$ Physiol (Lond) 1996;493:895-908.

30 Wessberg J, Kakuda N. Single motor unit activity in relation to pulsatile motor output in human finger movements. $f$ Physiol (Lond) 1999;517:273-85.

31 Elble RJ. Physiologic and essential tremor. Neurology 1986; 36:225-31.

32 Freund HJ, Dietz V. The relationship between physiological and pathological tremor. In: Desmedt JE, ed. Progress in clinical neurophysiology. Basel: Karger, 1978:66-89. 